\title{
FEATURES OF WEAKLY NONLINEAR WAVE INTERACTION
}

\author{
V.A. Buts ${ }^{1,2.3}$, I.K. Kovalchuk ${ }^{1}$, A.P. Tolstoluzhsky ${ }^{1}$ \\ ${ }^{1}$ National Science Center "Kharkiv Institute of Physics and Technology”, Kharkiv, Ukraine; \\ ${ }^{2}$ V.N. Karazin Kharkiv National University, Kharkiv, Ukraine; \\ ${ }^{3}$ Institute of Radio Astronomy of NAS of Ukraine, Kharkiv \\ E-mail: tolstoluzhsky@kipt.kharkov.ua
}

Regular and stochastic dynamics of three wave interaction and dynamics of cascades of such three wave processes were investigated. It was shown that matrix elements of wave interaction are inversely proportional to square of frequency of LF wave participating in three wave interaction. It was shown, that thresholds of arising of regimes with dynamics chaos are proportional to cube of LF wave. By numerical methods dynamics of cascades of interacting waves was investigated in detail.

PACS: 52.35.Mw

\section{INTRODUCTION}

Decay processes in plasma are well studied example of weakly nonlinear interaction of wave. Their results are widely presented in the scientific literature. The fullest they are presented, for example, in [1 - 3]. In works $[4,5]$ it was shown that there are conditions when decay process may transfer into stochastic regime. Stochastic decays were investigated as numerically as experimentally. Results of these investigations are presented in works [6 - 12].

This process may be continued. As result, the cascade of decays is received. One of the variants of such process is presented in [13]. It may talk about cascades with different scenarios. In one of such cases the low frequency wave is common for all decays. In other case in each of cascade decay different low frequency waves participate [14].

The one three wave decay is well have investigated. Hower, as result of one three wave decay the waves may appear that self may decay. In some cases there can be many such elementary wave processes. There is natural question about redistribution wave energy in such cascade of decays. Essential interest for plasma physics in this case is question about energy value that is transferred into low frequency part of spectra that well interacts with ion component of plasma. Besides, it is important to find conditions when dynamics of these low frequency waves is chaotic.

It should be noted that in the overwhelming majority of studies of the processes of nonlinear interaction of waves, dimensionless both dependent and independent variables are mainly used. In many cases it is convenient. However, often with this consideration, the functional dependence of the studied processes on the system parameters is lost.

\section{DYNAMICS OF ISOLATED THREE- WAVE INTERACTION}

Dynamics of amplitudes of interacting waves at three wave interaction is described by set of equations that contained in works [1 - 3].

$$
\frac{\partial a_{1}}{\partial t}=V a_{2} a_{3} e^{i \delta t}, \frac{\partial a_{2}}{\partial t}=-V a_{1} a_{3}^{*} e^{-i \delta t}, \frac{\partial a_{3}}{\partial t}=-V a_{1} a_{2}^{*} e^{-i \delta t},
$$

where $a_{i}$ - slowly varying amplitudes of interacting mode; $\delta=\omega_{1}-\omega_{2}-\omega_{3}-$ detuning; $\omega_{1,2}-$ frequencies of HF waves; $\omega_{3}$ - frequency of LF wave; $V$ - matrix elements of nonlinear wave interaction.

In this section we will investigate strict synchronism. In this case is convenient to use dimensionless time $\tau=V t$. Set of equation (1) is universal. Hower, it is important to define dependence of matrix element versus LF wave frequency

To find this dependence from the Maxwell equations, we write out the following relationship of the electric field strength with a non-linear current:

$$
\frac{\partial E_{1}}{\partial t}=-\frac{4 \pi \omega_{1}}{c^{2}} \frac{\vec{e}_{1}^{*} \vec{j}_{n l 1}}{A_{1}},
$$

where $E_{1}$ - amplitude of decaying wave in the CGS system (in contrast of $a_{1}$ - which is normalized correspondingly method that is presented in [1]);c-speed of light; $\vec{e}_{1}$ - polarization vector of decaying wave; $\vec{j}_{n l 1}-$ density of nonlinear current; $A_{1}$ - value that functionally depend versus frequency and wave vector of decaying wave.

Note that equation (2) already is shortened. The expression for density of nonlinear current can be obtained by means equation of hydrodynamics for electron and ions and has next form:

$$
\vec{j}_{n l 1}^{(1)}=-e\left(\tilde{n}_{e 2} \tilde{v}_{e 3}\right)-e\left(\tilde{n}_{e 3} \tilde{v}_{e 2}\right),
$$

where $e$ - electron charge; $\tilde{n}_{e j}, \tilde{v}_{e j}$-disturbance of electron density and velocity correspondingly in the field of $j$-th wave and have next form:

$$
\tilde{n}_{e 3}=-i \frac{n_{0} e k_{z 3}}{m_{e} \omega_{3}^{2}} E_{z 3}, \quad \tilde{v}_{e z 2}=-i \frac{e e_{z 2}}{m_{e} \omega_{2}} E_{z 2} .
$$

Substituting expressions (4) into (3) and last in (2) it is obtained expression that is analogous to the first equation of set (1)

$$
\frac{\partial E_{1}}{\partial t}=V_{1} E_{2} E_{3}
$$

where $V_{1}$ is:

$$
V_{1}=\frac{\omega_{p e}^{2} e k_{3}}{m_{e} c^{2} A_{1}} \frac{1}{\omega_{3}^{2}} .
$$

Formula (6) explicitly expresses dependence of matrix element versus parameters of low frequency wave. Namely, explicit dependence versus frequency of LF wave is visible $\left(V \approx 1 / \omega_{3}^{2}\right)$. 


\subsection{CONDITIONS OF ARISING REGIMES WITH DYNAMIC CHAOS}

We will show in this subsection that the conditions of the transition to the regime with dynamic chaos depend anomalously on the frequency of the lowfrequency wave participating in the interaction. Namely, the criterion is inversely proportional to the cube of this frequency. Indeed, it was shown in $[4,5]$ that the decaying process can go into the stochastic regime if the increment of the decaying instability $\left(V a_{10}, a_{10}\right.$ is the initial amplitude of the decaying wave) exceeds the frequency of the most low-frequency wave. This criterion is:

$$
\frac{V a_{10}}{\omega_{3}} \approx \frac{a_{10}}{\omega_{3}^{3}}>1 .
$$

From (7) it follows that stochastic regimes in decays can most easily be realized with the participation of waves with low frequencies. This circumstance can be used, for example, to heat the ion component of the plasma.

\subsection{DEPENDENCE OF THE WAVES DYNAMICS INTERACTION ON THE VALUE OF DETUNING}

Above, a rather idealized case was considered when the characteristics of interacting waves strictly satisfy the synchronism conditions. If these conditions are not satisfied that it is necessary to analyze the system of equations (1)

At the initial stage of decay, we will assume the amplitude of the pumping wave $a_{1}=a_{10}=$ const . Representing a solution for the low-frequency wave in the form $a_{3}=a_{30} e^{i \omega t}$ we find the dispersion equation

$$
\omega(\omega+\delta)=-|V|^{2}\left|a_{10}\right|^{2} \text {, }
$$

whose solution has the form

$$
\omega_{1,2}=\left(-\delta \pm \sqrt{\delta^{2}-|V|^{2}\left|a_{10}\right|^{2}}\right) / 2 \text {. }
$$

Thus, in the presence of detuning, at the initial stage, the unstable dynamics of decay occurs under the condition $\delta^{2}-|V|^{2}\left|a_{10}\right|^{2}<0$ that it is of a threshold nature. With strict synchronism ( $\delta=0)$, the dynamics have the character of instability with an increment $\gamma=\operatorname{Im}(\omega)=|V|\left|a_{10}\right|$.

\subsection{ACCOUNTING WAVE PROPERTIES OF LOW-FREQUENCY WAVE}

In the previous subsection the case was studied when the wave amplitude dynamics was considered only on the basis of shortened equations for all the waves involved in the interaction. In many cases, the frequency of the low-frequency wave can be quite small, so that the characteristic times of non-linear interaction of waves are on the order of the low-frequency wave period. In this case, the wave properties of lowfrequency waves can significantly affect the dynamics of the interaction of waves. In this subsection, we will take into account this possibility.

In this case, the system of equations (1) should be rewritten:

$$
\begin{gathered}
i \frac{d a_{0}}{d t}=V b a_{-1} e^{i \Delta t}, \quad i \frac{d a_{1}}{d t}=V b^{*} a_{0} e^{-i \Delta t}, \\
\frac{d^{2} b}{d t^{2}}+\Omega^{2} b=-2 \Omega V a_{-1}^{*} a_{0} e^{-i \Delta t},
\end{gathered}
$$

where $\Delta=\omega_{0}-\omega_{-1}, \quad \Omega=\omega_{3}$.

At the initial stage of decay, we will also assume the amplitude of the pump wave $a_{0}=$ const. Writing the solution for the low-frequency wave we write in the form $b=b_{0} e^{i \omega t}$, we obtain the dispersion equation:

$$
\left(\omega^{2}-\Omega^{2}\right)(\Delta+\omega)-2 \Omega\left(|V|^{2}|a 0|^{2}\right)=0 .
$$

The analytical representation of solutions of the dispersion equation is bulky, therefore the numerical analysis of the solutions of this equation was carried out. The graph below presents the main features of the solutions of this equation (Fig. 1).
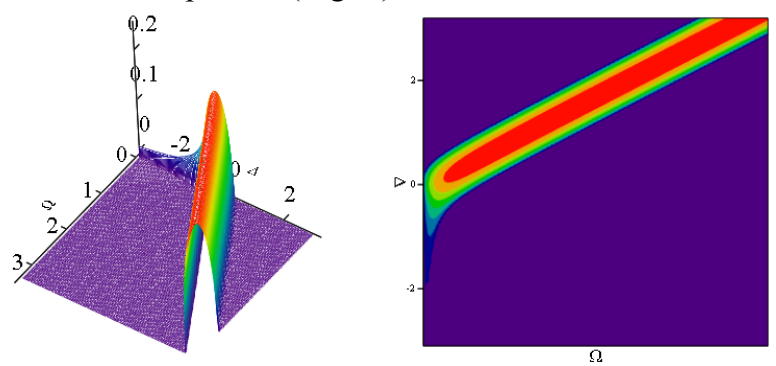

Fig. 1. The dependence of increment of the three-wave interaction on the parameters $\Delta$ and $\Omega$ for the pump wave amplitude $a_{0}=0.25$

The maximum value of the increment is directly proportional to the amplitude of the pumping wave.

With decreasing of the amplitude of the pumping wave, the region of frequencies and detunings, at which there exist instability-like regimes, narrows and for small values of the pumping wave amplitude $a_{0} \leq 0.1$, the instability develops under conditions close to resonant: $\Delta-\Omega \cong 0$.

The transition to stochasticity has the form

$$
\frac{\left(V a_{10}\right)}{\omega_{3}}>1 \text {. }
$$

Note that the frequencies and wave vectors of the interacting waves must satisfy the synchronism conditions $(\delta=0)$.

\section{DYNAMICS OF LIMITED NUMBER OF ELEMENTARY THREE-WAVE PROCESSES}

For the analysis of Dynamics of the limited cascade of decays, it is convenient to use the following system of equations:

$$
\begin{aligned}
& i \frac{d a_{n}}{d t}=V_{n-1} b a_{n-1} e^{i \delta t}+V_{n+1} b^{*} a_{n+1} e^{-i \delta t}, \\
& i \frac{d b}{d t}=\sum_{n=-N_{1}}^{N_{2}} V_{n-1} a_{n-1}^{*} a_{n} e^{-i \delta t} .
\end{aligned}
$$

Here $\delta=\omega_{n}-\omega_{n-1}-\Omega$ is detuning, $\omega_{n}$ are frequencies of the high-frequency wave, $\Omega$ is frequency of the low-frequency wave, $V_{n}$ are the matrix elements of the nonlinear interaction of the waves. At the same time, we assume that the frequencies and wave vectors of high- 
frequency waves are much higher than the frequencies and wave vectors of low-frequency waves $\omega>>\Omega, k>>$.

Under this condition, the matrix elements $V_{n}$ weakly depend on the number $n$. We will consider them the same and equal to one.

In real systems, the number of high-frequency interacting waves, although large, but is finite. Therefore, we will assume that the amplitudes of the waves $a_{n}=0$, if $n$ it is outside the range of values $-N_{1} \leq n \leq N_{2}\left(N_{1}\right.$ is number of red satellites, $N_{2}$ is number of blue satellites). The system of equations (14) up to terms of order $\left(\Omega / \omega_{0}, \kappa / k\right)$ has the integrals of motion:

$$
\begin{gathered}
\sum_{n=-N_{1}}^{N 2} \omega_{n}\left|a_{n}\right|^{2}+\Omega|b|^{2}=\varepsilon, \\
\sum\left|a_{n}\right|^{2}=I .
\end{gathered}
$$

The relation (14) represents the total energy of the interacting waves, and the relation (15) can be interpreted as the number of quanta of the interacting highfrequency waves. Besides, it is possible to show that

$$
\frac{d}{d t} \sum_{n=-N_{1}}^{N_{2}} a_{n-1}^{*} a_{n}=\left(\left|a_{-N_{1}}\right|^{2}-\left|a_{N_{2}}\right|^{2}\right) \text {. }
$$

And in the case when the number of "red" and "blue" satellites is equal $\left(N_{1}=N_{2}\right)$, under the initial conditions $\left(a_{0}^{0}=a_{0}(t=0), b 0=b(t=0)\right)$ one more integral of motion takes place:

$$
\sum_{n=-N_{1}}^{N_{2}} a_{n-1}^{*} a_{n}=\text { const }
$$

\subsection{NUMERICAL ANALYSIS}

Analytical study of system (13) is possible only for an infinite number of interacting waves (see [1]). To analyze the dynamics of a finite number of interacting waves, it is necessary to use numerical methods. The system of equations (13) was solved numerically under various initial conditions for the fields and different detuning parameters $\delta$. We investigated the temporal dynamics of wave interaction, the power spectra $(S(\omega))$ of realizations, their autocorrelation functions $\left(\mathrm{C}_{\mathrm{f}}\right)$, and the maximum Lyapunov exponent of the system $(\sigma)$.

At the initial moment of time, the real values of the fields were chosen: $\operatorname{Re} a_{0}(t=0)=a_{0}^{0} ; \operatorname{Re} a_{-1}(\mathrm{t}=0)=a_{-1}^{0}$; $b(t=0)=b_{0}$. The imaginary parts of the fields and the amplitudes of other waves at $t=0$ were selected equal to zero. The accuracy of the calculations was controlled by means of integral (16). The magnitude of the deviation from the integral

$$
\Delta_{\text {int }}=\sum\left|a_{n}(t)\right|^{2}-\sum\left|a_{n}(t=0)\right|^{2} \leq 10^{-9}
$$

in all conducted numerical researches.

In Fig. 2 results of numerical calculations of system (13) are shown for an asymmetric case ( $N_{1}=7, N_{2}=0$ ) with the initial conditions of the beatwave type $a_{0}^{0}=1$ and $a_{-1}^{0}=0.1$, when synchronism condition ( $\delta=0$ ) is fulfilled.
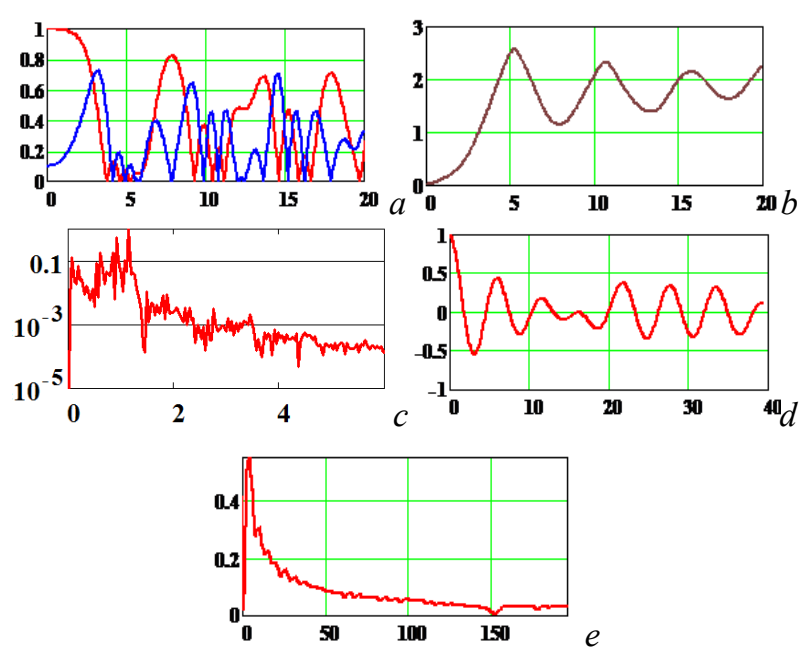

Fig. 2. Dependences of magnitudes of amplitude on time: a) amplitude $a_{0}$ is red line, amplitude $a_{-1}$ is blue line; b) amplitude $b ; c, d)$ spectrum and autocorrelation function of amplitude;

e) $\sigma$ is the maximum Lyapunov index

From these graphs it can be seen that the amplitudes of high-frequency oscillations have the form corresponding to the analytical representation of oscillations [14] at time intervals up to $t^{*} \cong 5.1$, after which a complicated dynamics of the amplitude of interacting waves occurs.

A peculiarity of this type of nonlinear wave interaction is the fact that the number of low-frequency wave quanta increases over the time interval when the disturbance did not reach the last waves participating in the interaction, after which oscillations of the number of low-frequency wave quanta occur. The maximum number of low-frequency quanta $N_{b}=|b|^{2}$ in this case exceeds the number of quanta of each of the highfrequency waves $N_{k}=\left|a_{k}\right|^{2}$ by more than six times. The maximum Lyapunov index of the system does not exceed in order of magnitude $\sigma<2 \cdot 10^{-2}$; therefore, in this case, we can speak about regular dynamics of the decay cascade.

As the number of interacting waves increases $N_{1}=11, N_{2}=0$, as shown by numerical analysis, the maximum amplitude of the low-frequency wave increases and time increases somewhat $t^{*} \cong 6.0$. In this case, if the initial amplitudes of the waves are increased $a_{0}^{0}=1$ and $a_{-1}^{0}=0.5$, the maximum amplitude of the low-frequency wave increases and time decreases $t^{*} \cong 4$. Other characteristics of the decay process of the waves at the same time qualitatively change a little.

The results of numerical calculations for the symmetric case $\left(N_{1}=N_{2}=7\right)$ with initial conditions of the beat-wave type $a_{0}^{0}=1$ and $a_{-1}^{0}=0.1$, when the synchronism condition ( $\delta=0)$ is satisfied, show that the amplitudes of high-frequency oscillations also have the form corresponding to the analytical representation oscillations [14] on the time intervals necessary for the disturbance to reach the last waves involved in the interaction. 
The spectrum of the low-frequency wave line, the correlation function is periodic. The maximum Lyapunov exponent $\sigma$ decreases with time and in order of magnitude $\sigma<0.02$, so that one can speak to the regular dynamics of the waves.

A feature of this type of nonlinear wave interaction is the fact that the number of low-frequency wave quanta increases linearly at time intervals $t^{*} \cong 10$ when the disturbance does not reach the last waves participating in the interaction, after which it falls linearly to zero and the dynamics of quanta of low-frequency waves repeats. The maximum number of low-frequency quanta does not exceed the number of high-frequency quanta.

\section{DYNAMICS OF THE LIMITED CASCADE TAKING INTO ACCOUNT WAVE PROPERTIES OF THE LOW-FREQUENCY WAVE}

Since the frequency of the low-frequency wave is rather small $\left(\omega_{n}>>\Omega\right)$ when averaging its equation over a high frequency, it is necessary to use not a shortened equation, but a second-order equation.

At the same time the system of the equations, necessary for the analysis, takes a form:

$$
\begin{aligned}
& i \frac{d a_{n}}{d t}=b a_{n-1} e^{i \Delta t}+b^{*} a_{n+1} e^{-i \Delta t}, \\
& \frac{d^{2} b}{d t^{2}}+\Omega^{2} b=-2 \Omega \sum_{n=-N_{1}}^{N_{2}} a_{n-1}^{*} a_{n} e^{-i \Delta t},
\end{aligned}
$$

where $\Delta=\delta+\Omega=\omega_{n}-\omega_{n-1}$.

\subsection{NUMERICAL ANALYSIS}

For a finite number $n$, the system of equations (18), also as well as in the previous case, was solved numerically under different initial conditions for the fields and different values of detuning $\Delta$ and frequency $\Omega$. At the same time, at the initial moment of time, the real values of the fields were chosen: $\operatorname{Re} a_{0}(\mathrm{t}=0)=a_{0}^{0} ; \operatorname{Re} a_{-1}(\mathrm{t}=0)=a$. ${ }_{1}^{0} ; b(t=0)=b_{0}$. The imaginary parts of the fields and the amplitudes of the other waves at $t=0$ were chosen to be zero. The value of the deviation from the integral (15) $\Delta_{\text {int }} \leq 10^{-9}$.

Below are the results of a numerical analysis of the system of equations (18).

In Fig. 3 results of numerical calculations of system (18) for an asymmetric case $\left(N_{1}=7, N_{2}=0\right)$ with initial conditions of the beat-wave type $a_{0}^{0}=1$ and $a_{-1}^{0}$ $=0.1-$ are shown, at $\Omega=0.3, \Delta=0.3$.

From these graphs it can be seen that at the beginning of the interaction, the amplitudes of high-frequency oscillations also have the form corresponding to the analytical representation oscillations [14] on the time intervals necessary for the disturbance to reach the last waves involved in the interaction. After that, the amplitudes of high-frequency oscillations have a shape similar to irregular oscillations with different intensities, whose frequencies also change irregularly.

The spectra of these waves take the form of a "table" type; the correlation function rapidly decreases with time to zero value and has irregular oscillations near this value. Therefore, we can speak about random amplitude and frequency modulation of HF waves. The spectrum of the low-frequency wave is is a little widened, correlation function slowly decreases remaining periodic, so that the dynamics of the low-frequency wave is regular. The maximum Lyapunov exponent eventually reaches almost a constant level $\sigma \sim 0.2$ (see Fig. 3,g), that confirms the chaotic nature of the process. Consequently, when a low-frequency wave interacts with a system of high-frequency waves with chaotic dynamics, the dynamics of the low-frequency wave remains regular.
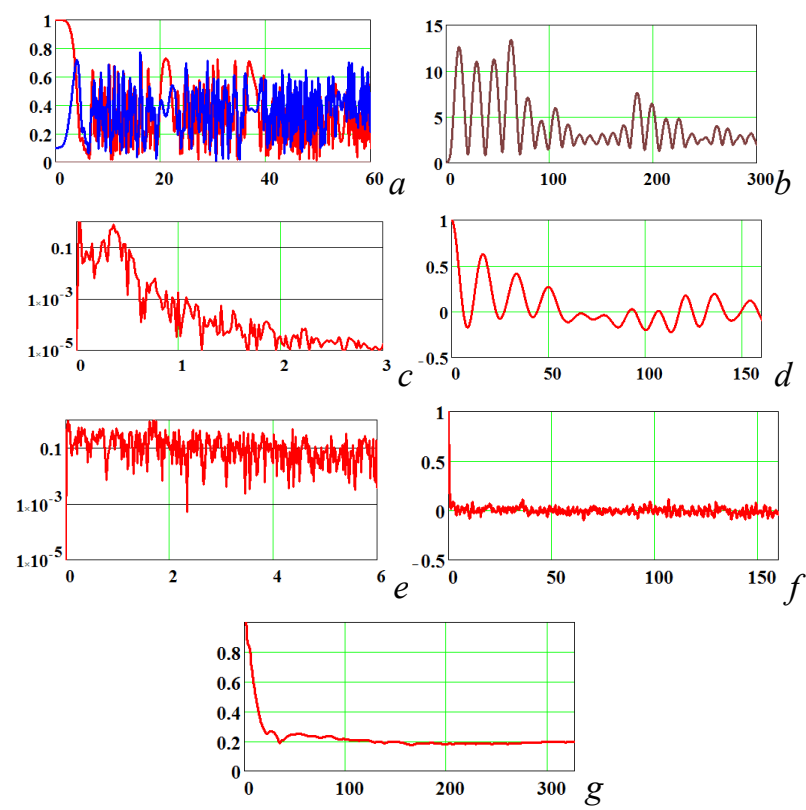

Fig. 3. The magnitudes of the amplitude on time are a) amplitude $a_{0}$ is red line, amplitude $a_{-1}$ is blue line;

b) amplitude $b ; c, d)$ spectrum and autocorrelation function of the amplitude of the low frequency wave; $e, f)$ spectrum and autocorrelation function of the amplitude of the high frequency wave; $g$ ) is the maximum

\section{Lyapunov exponent $\sigma$}

The number of quanta of the low-frequency wave grows on time interval when the perturbation has not reached the last waves participating in the interaction, after which oscillations of the number of low-frequency quanta occur. The maximum number of low-frequency quanta $N_{b}=|b|^{2}$ exceeds the number of quanta of each of the high-frequency waves $N_{k}=\left|a_{k}\right|^{2}$ by more than an order of magnitude.

Increase the number of waves involved in the cascade does not lead to significant change in the dynamics of the interaction of waves.

In the symmetric case $\left(N_{1}=N_{2}=7\right)$ with $a_{0}^{0}=1$ and $b^{0}=b(t=0)=0.1$, when $\Omega=0.3$ and $\Delta=0.3$ the lowfrequency wave spectrum is line, the autocorrelation function changes periodically with time, the pump wave spectrum has linear character, the autocorrelation function slowly changes with time. The maximum Lyapunov index of the wave system $\sigma$ decreases with time to values $\sigma<1 \cdot 10^{-2}$, therefore in this case it is possible to say that dynamics of amplitudes as high frequency waves and low-frequency wave has regular character. 


\section{CONCLUSIONS}

Let's note the most important results:

1. The effective transformation of the energy of the high-frequency wave into the energy of the lowfrequency wave is possible at nonlinear wave interaction.

2. The magnitude of the matrix element of the nonlinear interaction of the waves is inversely proportional to the square of the low frequency. As a result, the process of decay to low frequencies occurs at lower field strengths of the decaying wave.

3. Modes with stochastic dynamics arise, primarily with the participation of the wave with a minimum frequency of the low-frequency waves. This is due to the fact that the threshold of stochastic instability is inversely proportional to the cube of low frequency.

4. The feature of the cascade of decays in which were took into account the wave properties of a low-frequency wave is that the maximum number of low-frequency wave quanta exceeds the number of quanta of each of the RF waves by more than an order of magnitude.

\section{REFERENCES}

1. B.B. Kadomtsev. Collective phenomena in plasma. M.: "Science". Gl. Red. Phis.-mat. Lit., 1988 (in Russian).

2. H. Wilhelmsson, J. Weiland. Coherent non-linear interaction of waves in plasmas. M.: "Energoatomizdat", 1981 (in Russian).

3. V.N. Tsitovich. Nonlinear effects in plasm. M.: "Science", 1967.

4. V.A. Buts, O.V. Manujlenko, K.N. Stepanov, A.P. Tolstoluzhsky. Chaotic dynamics of charged particles at wave-particle type interaction and chaotic dynamics at weak nonlinear interaction of wavewave type // Plasma physics. 1995, v. 20, № 9, p. 794-801 (in Russian).

5. V.A. Buts, A.N. Kupriyanov, O.V. Manujlenko, A.P. Tolstoluzhsky. Instability and dynamics chaos at weakly nonlinear interaction of waves // Izvestiya vuzov “PHD”. 1993, v. 1, № 1, 2, p. 57-62 (in Russian).

6. V.A. Buts, O.V. Manujlenko, K.N. Stepanov, A.P. Tolstoluzhsky. Chaotic dynamics of charged particles at wave-particle type interaction and chaotic dynamics at weak nonlinear interaction of wavewave type // Plasma Physics. 1995, v. 20, № 9, p. 794-801.

7. A.N. Antonov, V.A. Buts, I.K. Kovalchuk, O.F. Kovpik, E.A. Kornilov, V.G. Svichensky, D.V. Tarasov. Regular and Stochastic decays of Waves in a Plasma Cavity // Plasma Physics Re-ports. 2012, v. 38, № 8, p. 636-650.

8. A.N. Antonov, V.A. Buts, I.K. Kovalchuk, O.F. Kovpik, E.A. Kornilov, V.G. Svichensky, D.V. Tarasov. Investigation of chaotic decay in the resonator filled with plasma // Problems of Atomic Science and Technology. Series “Plasma Physics”. 2011, № 1, p. 89-91.

9. V.A. Buts, I.K. Kovalchuk. Dynamics of three wave stochastic decays in nonlinear matter // Problems of Atomic Science and Technology. Series "Plasma Physics”. 2016, № 6, p. 156-159.

10. A.N. Antonov, V.A. Buts, I.K. Kovalchuk, O.F. Kovpik, E.A. Kornilov, V.G. Svichensky, D.V. Tarasov. The peculiarities stochastic heating of the plasma in plasma resonator // Problems of Atomic Science and Technology. Series “Plasma Physcs”. 2014, № 6, p. 87-90.

11. V.A. Buts, I.K. Kovalchuk. Dynamics of three wave stochastic decays in nonlinear matter // Problems of Atomic Science and Technology. Series "Plasma Physics”. 2016, № 6, p. 156-159.

12. V.A. Buts, I.K. Kovalchuk, D.V. Tarasov, A.P. Tolstoluzhsky. Peculiarity of chaotic and regular dynamics of waves. 2012. http://arxiv.org/abc/ 1210.6788 .

13. V.A. Buts, I.K. Kovalchuk. Features of three wave interaction in the magnetoactive plasma // Problems of Atomic Science and Technology. Series "Plasma Physics”. 2015, № 1, p. 152-155.

14. A.S. Bakai // Nucl. Fusions. 1970, v. 10, p. 53.

15. V.A. Buts, I.K. Kovalchuk, D.V. Tarasov, A.P. Tolstoluzhsky. Regular and chaotic dynamics of weakly nonlinear wave interaction // Electromagnetic waves and electron systems. 2011, v. 16, № 1, p. 51-61 (in Russian).

Article received 04.06.2019

\section{ОСОБЕННОСТИ СЛАБОНЕЛИНЕЙНОГО ВЗАИМОДЕЙСТВИЯ ВОЛН \\ В.А. Буц, И.К. Ковальчук, А.П. Толстолужский}

Исследована регулярная и стохастическая динамика трехволнового взаимодействия, а также динамика каскадов таких трехволновых процессов. Показано, что матричные элементы взаимодействия волн обратно пропорциональны квадрату частоты НЧ-волны, участвующей в трехволновом взаимодействии. Показано, что пороги возникновения режимов с динамическим хаосом пропорциональны кубу частоты НЧ-волны. Численными методами детально исследована динамика каскадов взаимодействующих волн.

\section{ОСОБЛИВОСТІ СЛАБОНЕЛІНІЙНОЇ ВЗАЄМОДІЇ ХВИЛЬ}

\section{В.О. Буц, І.К. Ковальчук, О.П. Толстолужсський}

Досліджена регулярна та стохастична динаміка трихвилевої взаємодії, а також динаміка каскадів таких трихвилевих процесів. Показано, що матричні елементи взаємодії хвиль обернено пропорційні квадрату частоти НЧ-хвилі, що бере участь у трихвилевій взаємодії. Показано, що пороги виникнення режимів з динамічним хаосом пропорційні кубу частоти НЧ-хвилі. Чисельними методами детально досліджена динаміка каскадів хвиль, що взаємодіють. 